\title{
Research on nonlinear optical materials: an assessment
}

\author{
D. H. Auston, A. A. Ballman, P. Bhattacharya, G. J. Bjorklund, C. Bowden, R. W. Boyd, P. S. Brody, \\ R. Burnham, R. L. Byer, G. Carter, D. Chemla, M. Dagenais, G. Dohler, U. Efron, D. Eimerl, \\ R. S. Feigelson, J. Feinberg, B. J. Feldman, A. F. Garito, E. M. Garmire, H. M. Gibbs, A. M. Glass, \\ L. S. Goldberg, R. L. Gunshor, T. K. Gustafson, R. W. Hellwarth, A. E. Kaplan, P. L. Kelley, \\ F. J. Leonberger, R. S. Lytel, A. Majerfeld, N. Menyuk, G. R. Meredith, R. R. Neurgaonkar, \\ N. G. Peyghambarian, P. Prasad, G. Rakuljic, Y.-R. Shen, P. W. Smith, J. Stamatoff, G. I. Stegeman, \\ G. Stillman, C. L. Tang, H. Temkin, M. Thakur, G. C. Valley, P. A. Wolff, and C. Woods
}

The seven papers making up this assessment are based on the Workshop on Nonlinear Optical Materials held in April 1986.

Received 1 October 1986.

0003-6935/87/010211-24\$02.00/0.

(C) 1987 Optical Society of America.

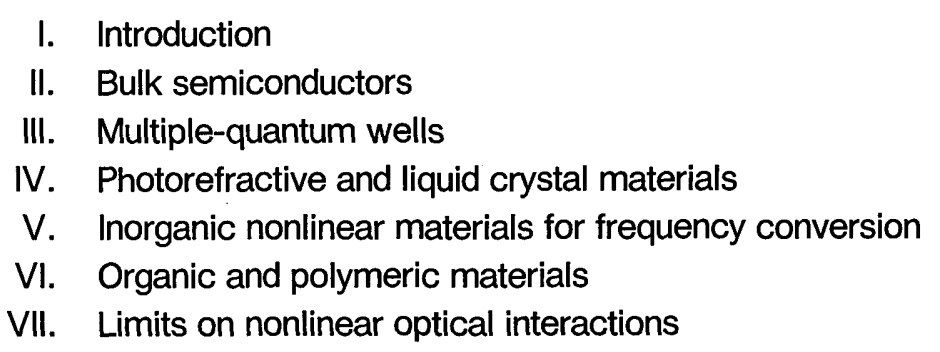

